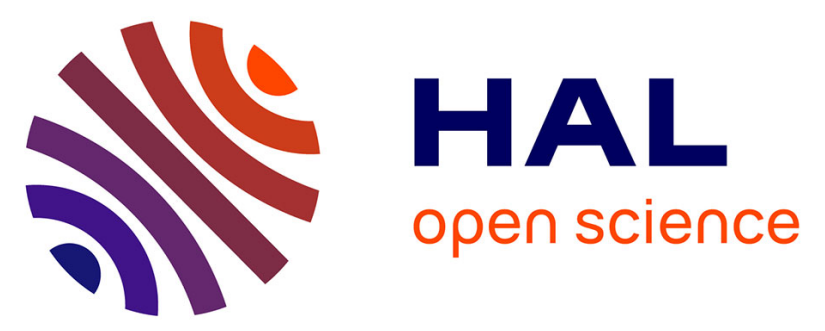

\title{
Kinematics, Design and Experimental Validation of a Novel Parallel Robot for Two-Fingered Dexterous Manipulation
}

Wissem Haouas, Guillaume Laurent, Sébastien Thibaud, Redwan Dahmouche

\section{- To cite this version:}

Wissem Haouas, Guillaume Laurent, Sébastien Thibaud, Redwan Dahmouche. Kinematics, Design and Experimental Validation of a Novel Parallel Robot for Two-Fingered Dexterous Manipulation. RSJ International Conference on Intelligent Robots and Systems, Nov 2019, Macau, China. 10.1109/IROS40897.2019.8968570 . hal-03427004

\section{HAL Id: hal-03427004 https://hal.science/hal-03427004}

Submitted on 12 Nov 2021

HAL is a multi-disciplinary open access archive for the deposit and dissemination of scientific research documents, whether they are published or not. The documents may come from teaching and research institutions in France or abroad, or from public or private research centers.
L'archive ouverte pluridisciplinaire HAL, est destinée au dépôt et à la diffusion de documents scientifiques de niveau recherche, publiés ou non, émanant des établissements d'enseignement et de recherche français ou étrangers, des laboratoires publics ou privés. 


\title{
Kinematics, Design and Experimental Validation of a Novel Parallel Robot for Two-Fingered Dexterous Manipulation
}

\author{
Wissem Haouas, Guillaume J. Laurent, Sébastien Thibaud and Redwan Dahmouche
}

\begin{abstract}
Two-fingered manipulation robotic systems are widely used in many applications and notably at small scales. The commonly employed solution is based on the attachment of a gripper on a robot. This paper introduces a new eight degrees of freedom (DoF) mechanism intended for two-fingered dexterous manipulation. The eight DoFs are obtained via a parallel architecture moved by eight actuators that are attached to the base of the robot. The novelty lies in the use of a 2-DoF configurable platform that insures two relative rotations of the gripper jaws (opening and twisting) in addition to the 6-DoF Cartesian movements. The paper presents the kinematics and the design of a proof-of-concept able to grasp, roll, translate and rotate objects in a single compact design. A prototype is operated to manipulate and insert a 2-mm screw.
\end{abstract}

\section{INTRODUCTION}

Serial manipulators have the advantage of sweeping large workspaces and dexterous maneuverability like human arms. However, their rigidity is low compared to parallel manipulators due to the cantilever structure. Thus, when high load carrying capacity and high precision are required, parallel robots are usually preferable [1]. They are also often used in industry for high-speed operations [2], [3]. However, their limited workspace and degrees of freedom (DoF) restrain their dexterity which makes them more suitable for simple tasks like pick-and-place operations [4], [5].

For applications where dexterity and precise positioning are requested, alternatives to conventional parallel manipulators must be found. Therefore, the general adopted solution in robotic manipulation is having an additional gripper attached to the end of a robot. However, having an extra gripper has some disadvantages like the weight of the tool that reduces the dynamic performances of the robot. Moreover some applications also require high compactness like robotic surgery and micro/nano manipulations where the manipulator evolves inside confined environments.

During the last decades, some parallel robots have been proposed with an additional DoF in the platform without compromising its parallel architecture [6]. It is achieved by replacing the rigid platform by a configurable one [7], [8]. This approach allows to integrate the grasping functionality in the same structure and to maintain the robot dynamics and compactness. In addition, no

The authors are with FEMTO-ST Institute, Univ. Bourgogne Franche-Comté, CNRS/UFC/ENSMM, Besançon, France. wiring or tubing to transmit power and signals is required to actuate the end-effector [9].

Relatively few parallel manipulators with configurable platforms have been proposed in the literature for grasping and manipulating applications. In 2015, Lambert proposed a redundantly actuated parallel haptic device with seven DoFs [10]. In [11] and [12] kinematic redundancy of novel parallel robots was exploited to enlarge the rotational workspace and remotely operate a gripper. Our team introduced, respectively in 2016 and 2018, a 4-DoF parallel spherical wrist with a foldable platform for minimally invasive surgery that generates the three rotations and the grasping/cutting function [13], [14] and a 7-DoF parallel manipulator with a foldable platform [15]. To perform dexterous micro-manipulation, a miniaturized version of the kinematic structure of the robot has been realized using a new micro-fabrication technique [16]. The common point between all these architectures is that the gripper has only one DoF which might limit the manipulation capabilities of the robot.

The objective of this work is to improve the dexterity of the manipulators proposed previously to achieve complex tasks in confined environments [17]-[19]. This paper introduces a new eight degrees of freedom (DoF) mechanism intended for two-fingered dexterous manipulation. The novelty of the proposed manipulator is the use of a 2-DoF configurable platform to insure the grasping and the rolling of objects between the gripper jaws. This rolling mobility offers large rotation angles of objects between the gripper fingers or the grasping of objects with different geometries. In addition, this capability can be used to provide the alignment of the fingers especially for some applications that need precise handling and insertion like in micro/nano-manipulation. This paper specially focuses on the concept and its experimental validation through a complex operation like inserting a screw with two fingers.

The paper begins with the presentation of the kinematics of the manipulator. Then, the inverse kinematics and the Jacobian matrix are developed. Finally, the design of a prototype and some experimental results are presented validating the novel kinematic structure.

\section{Kinematics of the Manipulator}

The proposed robotic structure is designed to grasp and roll objects between the gripper jaws while providing the 6-DoF to the gripper (rotations and translations). The motion of grasping and the rolling is obtained with 


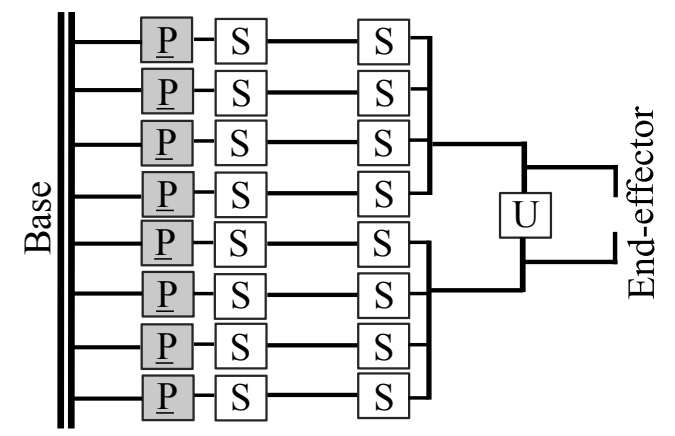

Fig. 1. Architectural scheme of the manipulator. The blocks $\mathrm{P}$, U, and $\mathrm{S}$ represent prismatic, universal, and spherical joints, respectively.

a configurable platform that insures both relative rotational DoF of the gripper jaws via a universal joint. Each part of the platform is linked two four linear actuators with four parallel limbs.

The result is a 8-DoF manipulator able to grasp, roll, position and rotate objects in a single compact design. The architectural scheme of the robot is illustrated in Fig. 1.

\section{A. Geometric Description}

The geometric model of the manipulator is presented in Fig. 2. This mechanism consists of eight identical kinematic chains. Each chain is composed, from base to the platform, of a linear actuator (that could be replaced by a rotational one), a spherical joint, a moving leg, and another spherical joint attached to a part of the configurable platform.

A fixed reference frame $\mathcal{F}_{w}=\left(O_{w}, \mathbf{x}_{w}, \mathbf{y}_{w}, \mathbf{z}_{w}\right)$ is attached to the base of the manipulator and a moving coordinate frame $\mathcal{F}_{p}=\left(O_{p}, \mathbf{x}_{p}, \mathbf{y}_{p}, \mathbf{z}_{p}\right)$ is placed at the center of the configurable platform (where the two axes associated to the universal joint cross) and when both parts of the platform are aligned.

Since the platform is composed of two parts linked via a universal joint, it has two independent relative rotations. In order to get the opening and the twisting of the gripper jaws, the rotational axis are chosen as a rotation around the $\mathbf{x}_{p}$ and $\mathbf{y}_{p}$ axis.

Thus, we define, respectively, ${ }^{p} \mathbf{R}_{r}$ and ${ }^{p} \mathbf{R}_{l}$ as the rotation matrices of the moving frames $\mathcal{F}_{r}$ and $\mathcal{F}_{l}$ attached respectively to the right and the left part of the platform as,

$$
\left\{\begin{array}{l}
{ }^{p} \mathbf{R}_{r}=\operatorname{rot}\left(\mathbf{x}_{p}, \theta\right) \cdot \operatorname{rot}\left(\mathbf{y}_{p}, \rho\right), \\
{ }^{p} \mathbf{R}_{l}=\operatorname{rot}\left(\mathbf{x}_{p},-\theta\right) \cdot \operatorname{rot}\left(\mathbf{y}_{p},-\rho\right),
\end{array}\right.
$$

where $\theta$ is the opening angle of the gripper around the $\mathbf{x}_{p}$ axis and $\rho$ is the twisting angle between both fingers of the gripper around the $\mathbf{y}_{p}$ axis.

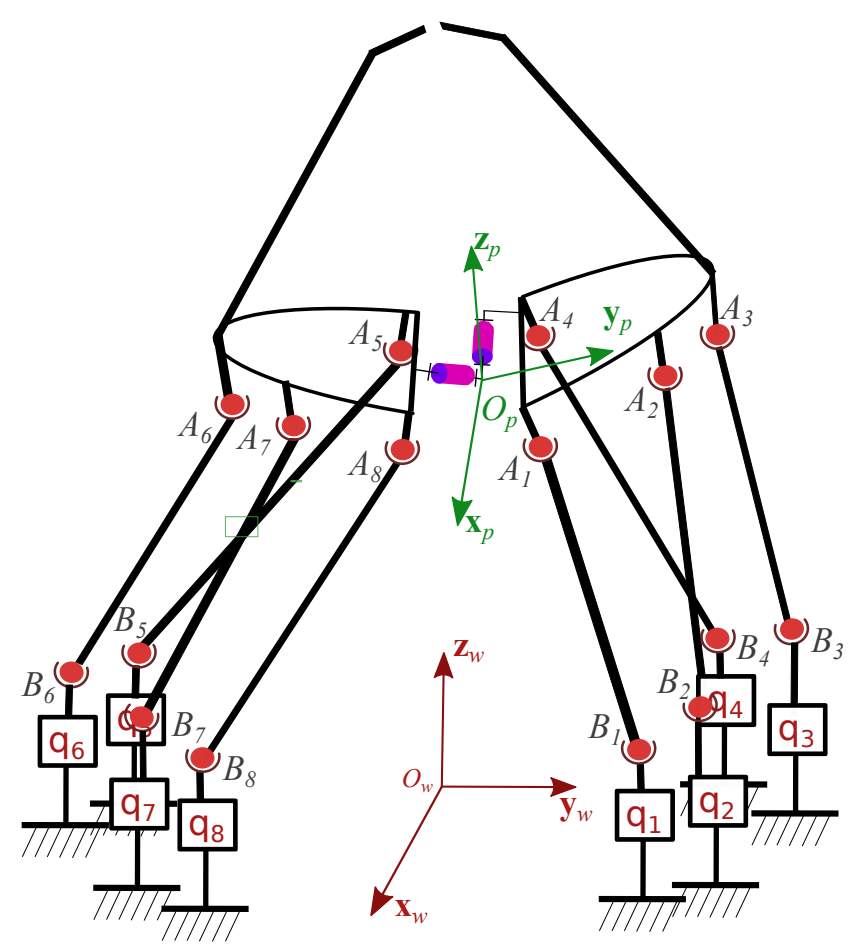

Fig. 2. Kinematics of the manipulator.

\section{B. Inverse Kinematics}

The aim of this section is to determine the eight joint positions that provide the desired end-effector pose.

The end-effector pose is defined by the coordinates of the point ${ }^{w} O_{p}$ (center of the platform) with respect to the fixed frame $\mathcal{F}_{w}$, by the orientation of the moving frame $\mathcal{F}_{p}$ represented by the rotation matrix ${ }^{w} \mathbf{R}_{p}$, by the opening angle $\theta$ and by the twist angle $\rho$.

The coordinates of the point $A_{i}$, the center of the spherical joint attached to the platform are given by,

$\left\{\begin{array}{l}{ }^{w} A_{i}={ }^{w} \mathbf{R}_{r}{ }^{r} A_{i}+{ }^{w} O_{p}={ }^{w} \mathbf{R}_{p}{ }^{p} \mathbf{R}_{r}{ }^{r} A_{i}+{ }^{w} O_{p}, \quad i=1, \ldots, 4 \\ { }^{w} A_{i}={ }^{w} \mathbf{R}_{l}{ }^{l} A_{i}+{ }^{w} O_{p}={ }^{w} \mathbf{R}_{p}{ }^{p} \mathbf{R}_{l}{ }^{l} A_{i}+{ }^{w} O_{p}, \quad i=5, \ldots, 8 .\end{array}\right.$

The actuators, in the chosen design, are linear and move vertically along the axis $\mathbf{z}_{w}$. Thus, the positions of the points $B_{i}$ with respect to the frame $\mathcal{F}_{w}$ are thus given by:

$$
{ }^{w} B_{i}={ }^{w} B_{0 i}+\left[\begin{array}{lll}
0 & 0 & q_{i}
\end{array}\right]^{t} \text { for } \quad i=1, \ldots, 8,
$$

with ${ }^{w} B_{0 i}$ representing the position of the point ${ }^{w} B_{i}$ in the reference configuration of the robot.

In order to solve the inverse kinematic problem, one must first consider the loop-closure constraint equations obtained from the length of each leg,

$$
\left\|^{w} B_{i}-{ }^{w} A_{i}\right\|=l_{i}, \quad i=1, \ldots, 8
$$


By replacing the equations 2 and 3 in 4 , the system of equations to be solved could be written as follows,

$\left(b_{0 x i}-a_{x i}\right)^{2}+\left(b_{0 y i}-a_{y i}\right)^{2}+\left(b_{0 z i}+q_{i}-a_{z i}\right)^{2}=l_{i}^{2}, \quad i=1, \ldots, 8$

where both solutions can express in the form,

$q_{i}=\left(a_{z i}-b_{0 z i}\right) \pm \sqrt{l_{i}^{2}-\left(b_{0 x i}-a_{x i}\right)^{2}-\left(b_{0 y i}-a_{y i}\right)^{2}}, i=1, \ldots, 8$

\section{Jacobian Matrix}

The Jacobian matrix could be obtained by taking the derivative of the kinematic Eq. 5 with respect to time. The system of equations can then be written in the form of a matrix multiplication,

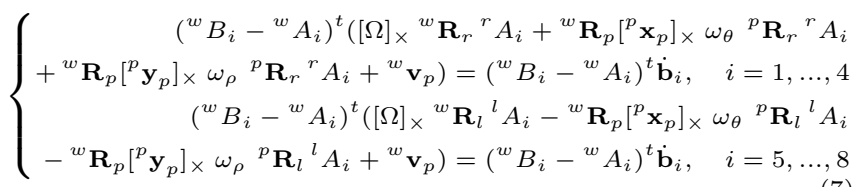

where, $\dot{\mathbf{b}}_{i}=\left[\begin{array}{lll}0 & 0 & \dot{q}_{i}\end{array}\right]^{t}$ is the instantaneous velocity of points ${ }^{w} B_{i}$, where $\dot{q}_{i}$ represents the vertical speed of an actuator. The vector $\Omega=\left[\begin{array}{lll}\omega_{\alpha} & \omega_{\beta} & \omega_{\gamma}\end{array}\right]^{t}$ is the instantaneous angular velocity of the frame ${ }^{w} \mathbf{R}_{p}$, and $\omega_{\theta}$ and $\omega_{\rho}$ are respectively the relatively instantaneous angular velocities between the two parts of the foldable platform in opening and twisting. The symbol $[\Omega]_{\times}$represents the cross product skew matrix, and ${ }^{w} \mathbf{v}_{p}=\left[\begin{array}{lll}v_{x} & v_{y} & v_{z}\end{array}\right]^{t}$ represents the velocity of the point $O_{p}$ located in the center of the foldable platform.

The system of equations 7 could be expressed in a matrix form as,

$$
\mathbf{J}_{l} \mathbf{t}=\mathbf{J}_{r} \dot{\mathbf{q}}
$$

here, $\mathbf{t}=\left[\begin{array}{llllll}\omega_{\alpha} \omega_{\beta} \omega_{\gamma} \omega_{\theta} \omega_{\rho} & v_{x} & v_{y} & v_{z}\end{array}\right]^{t}$ is the motion twist of the configurable platform and $\dot{\mathbf{q}}=$ $\left[\begin{array}{lllllllll}\dot{q}_{1} & \dot{q}_{2} & \dot{q}_{3} & \dot{q}_{4} & \dot{q}_{4} & \dot{q}_{5} & \dot{q}_{6} & \dot{q}_{7} & \dot{q}_{8}\end{array}\right]^{t}$ is the velocity of the linear actuators. The left matrix $\mathbf{J}_{l,(8 \times 8)}$ has the following form,

$\left[\begin{array}{cccc}\left(\overrightarrow{A_{1} B_{1}} \times \overrightarrow{O_{w} \overrightarrow{A_{1}}}\right)^{t} & \left(\overrightarrow{A_{1} B_{1}} \times \overrightarrow{O_{w} \overrightarrow{A_{1}}}\right) \cdot \mathbf{x}_{p} & \left(\overrightarrow{A_{1} B_{1}} \times \overrightarrow{O_{w} \overrightarrow{A_{1}}}\right) \cdot \mathbf{y}_{p} & \overrightarrow{A_{1} B_{1}} t \\ \left(\overrightarrow{A_{4} B_{4}} \times \overrightarrow{O_{w} \overrightarrow{A_{4}}}\right)^{t} & \left(\overrightarrow{A_{4} B_{4}} \times \overrightarrow{O_{w} \overrightarrow{A_{4}}}\right) \cdot \mathbf{x}_{p} & \left(\overrightarrow{A_{4} B_{4}} \times \overrightarrow{O_{w} \overrightarrow{A_{4}}}\right) \cdot \mathbf{y}_{p} & \overrightarrow{A_{4} B_{4}} t \\ \left(\overrightarrow{A_{5} \overrightarrow{B_{5}}} \times \overrightarrow{O_{w} \overrightarrow{A_{5}}}\right)^{t} & -\left(\overrightarrow{A_{5} B_{5}} \times \overrightarrow{O_{w} \overrightarrow{A_{5}}}\right) \cdot \mathbf{x}_{p} & -\left(\overrightarrow{A_{5} B_{5}} \times \overrightarrow{O_{w} \overrightarrow{A_{5}}}\right) \cdot \mathbf{y}_{p} & \overrightarrow{A_{5} B_{5}} t \\ \left(\overrightarrow{A_{8} \overrightarrow{B_{8}}} \times \overrightarrow{O_{w} \overrightarrow{A_{8}}}\right)^{t} & -\left(\overrightarrow{A_{8} B_{8}} \times \overrightarrow{O_{w} \overrightarrow{A_{8}}}\right) \cdot \mathbf{x}_{p} & -\left(\overrightarrow{A_{8} B_{8}} \times \overrightarrow{O_{w} \overrightarrow{A_{8}}}\right) \cdot \mathbf{y}_{p} & \overrightarrow{A_{8} B_{8}} t\end{array}\right]$

The right matrix $\mathbf{J}_{r,(8 \times 8)}$ could be expressed as follows,

$$
\mathbf{J}_{r}=\left[\begin{array}{ccc}
\overrightarrow{A_{1} B_{1}} \cdot \mathbf{z}_{w} & \cdots & 0 \\
\vdots & \ddots & \vdots \\
0 & \cdots & \overrightarrow{A_{8} B_{8} \cdot \mathbf{z}_{w}}
\end{array}\right]
$$

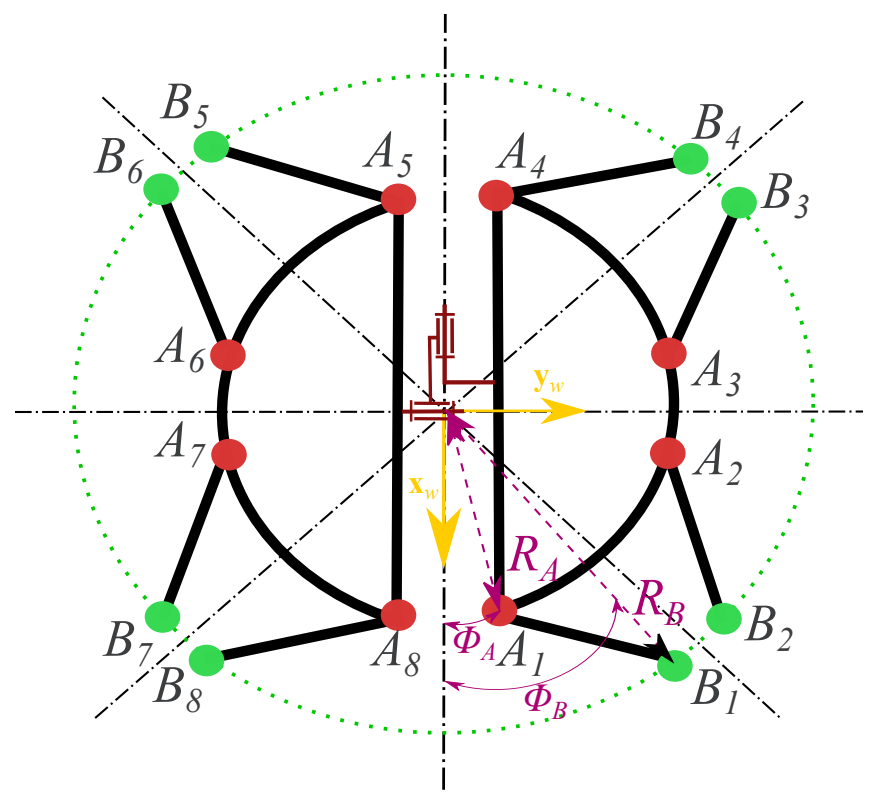

Fig. 3. Dimensional parameters of the structure (top-view).

where the $\times$ operator represents the cross products.

The Jacobian matrix relating the eight actuator velocities $\dot{\mathbf{q}}$ to the motion twist $\mathbf{t}$ is:

$$
\mathbf{t}=\mathbf{J}_{(8 \times 8)} \dot{\mathbf{q}}=\mathbf{J}_{l}^{-1} \mathbf{J}_{r} \dot{\mathbf{q}}
$$

This matrix is interesting for several reasons by the fact that it allows, to establish the direct and inverse kinematic model to control the speed of the robot (such as for visual servoing), to relate the forces exerted by the end-effector in correspondence to the forces/torques generated by the actuators, and to locate the singular configurations (when the determinant of $\mathbf{J}$ vanishes).

\section{Mechanical Design}

The kinematics properties of the structure have been developed as a function of its kinematic parameters in the previous section. The mechanical design of the prototype can now be chosen to obtain the required properties of a dexterous manipulator.

\section{A. Dimensional Parameters}

To balance the constraints and reduce the number of dimensional parameters, we consider a quaternary axial symmetry configuration formed of four pairs of identical kinematic chains arranged with a period of $90^{\circ}$. Each two legs are disposed symmetrically with respect to the plane formed by the axis $\mathbf{z}_{w}$ and $\operatorname{rot}\left(\mathbf{x}_{w}, j \frac{\pi}{4}+\frac{\pi}{8}\right)$, for $i=0, \ldots, 3$, and passing through the point $O_{w}$. The parametric lengths and the characteristic angles of this structure are represented in Fig. 3.

The dimensional parameters of the demonstrator, defined respectively in the reference configuration, are $h_{A_{i}}$ and $h_{B_{i}}$, the height of the coordinates ${ }^{w} A_{i}$ and ${ }^{w} B_{i}$, the radius $R_{A}$ and $R_{B}$ which are the radius of the circles passing through the joints ${ }^{w} A_{i}$ and ${ }^{w} B_{i}$, and finally $\phi_{A}$ 
TABLE I

Dimensional Parameters.

\begin{tabular}{|c|c|c|}
\hline & Parameter & Value \\
\hline $\mathrm{H}_{B}$ & Height of the $B_{i}$ joints & $80 \mathrm{~mm}$ \\
$\mathrm{H}_{A}$ & Height of the $A_{i}$ joints & $0 \mathrm{~mm}$ \\
$\mathrm{R}_{B}$ & Base radius & $45 \mathrm{~mm}$ \\
$\mathrm{R}_{A}$ & Platform radius & $19.5 \mathrm{~mm}$ \\
$\phi_{A}$ & Angle between $\mathbf{x}_{w}$ and $O_{w} A_{1}$ & $7.5^{\circ}$ \\
$\phi_{B}$ & Angle between $\mathbf{x}_{w}$ and $O_{w} B_{1}$ & $40^{\circ}$ \\
\hline
\end{tabular}

TABLE II

MAXimum DisPlaCEMENTS AND ROTATION ANGLES OF THE END-EFFECTOR AROUND THE REFERENCE POSE.

\begin{tabular}{|c|c|c|}
\hline & $\max$ & $\min$ \\
\hline$\alpha(\mathrm{deg})$ & 26 & -26 \\
$\beta(\mathrm{deg})$ & 26 & -26 \\
$\gamma(\mathrm{deg})$ & 52 & 52 \\
$\theta(\mathrm{deg})$ & 25 & -28 \\
$\rho(\mathrm{deg})$ & 25 & -25 \\
$x(\mathrm{~mm})$ & 18.5 & -18.5 \\
$y(\mathrm{~mm})$ & 18.5 & -18.5 \\
$z(\mathrm{~mm})$ & 9 & -9 \\
\hline
\end{tabular}

and $\phi_{B}$ which are respectively the angles between the line $\left(O_{w}, \mathbf{x}_{w}\right)$, and $\left(O_{w}, A_{1}\right)$ and $\left(O_{w}, B_{1}\right)$.

\section{B. Parameters Determination}

Since the prototype is highly symmetrical, we can expect relatively homogeneous performance inside the workspace. Nevertheless, due to the high number of DoF of the structure, it is not possible to consider a single performance criteria to determine the dimensional parameters. Since the aim of this paper is to build a first proof-of-concept, we used our know-how based on previous works [13]-[15] to design a well balanced manipulator able to achieve a screwing task.

The chosen dimensional parameters for the prototype are presented in Table I. The workspace of the manipulator using the selected kinematic parameters are presented in Table II. The different values were obtained by varying each coordinate of the end-effector pose independently and keeping others on zero. A MATLAB simulation was used to estimate the workspace of the manipulator by sampling the different parameters and taking into account a travel motion of the actuator of $18 \mathrm{~mm}$.

\section{Prototype Implementation}

This section describes the technical solutions adopted to fabricate a first prototype. These choices have been made to ensure the ease of its fabrication and assembly.

The design of the manipulator is presented in Fig. 4. For the legs, ball-and-socket joints were used to reduce

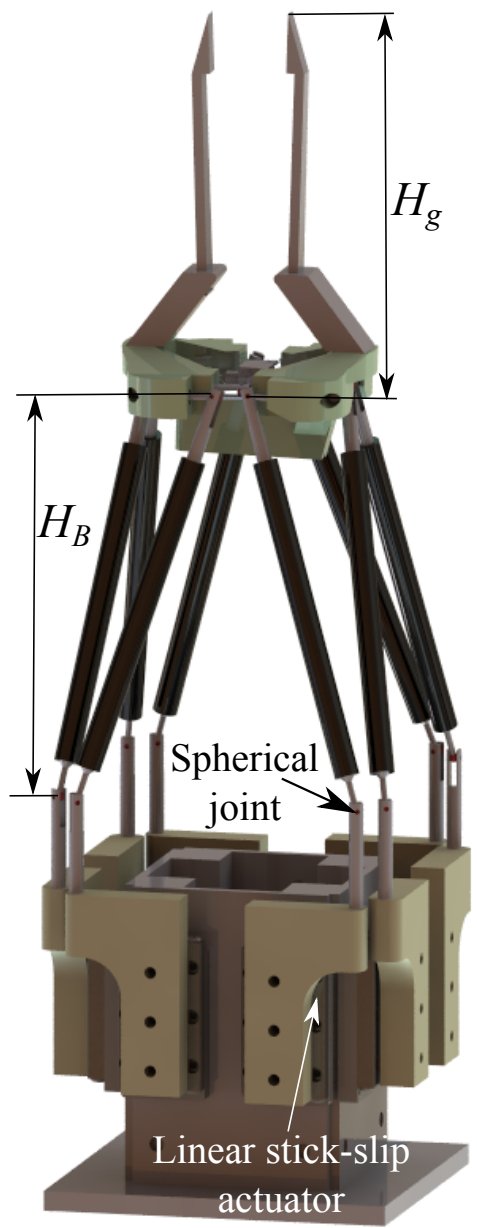

Fig. 4. Design of the prototype.

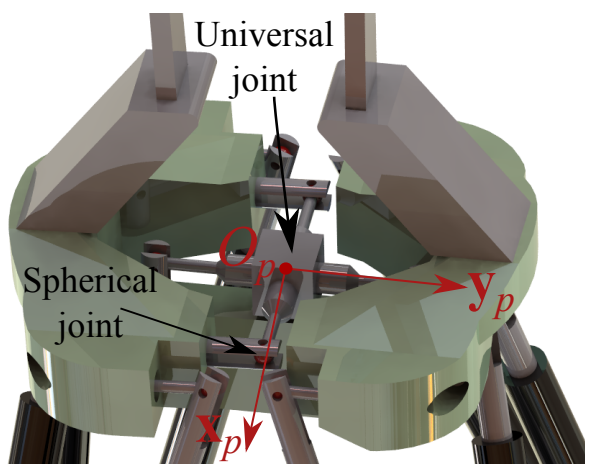

Fig. 5. Design of the configurable platform.

frictions and backlash. Each spherical ruby is inserted in a notch and held between coaxial through bores. The universal joint connecting the two parts of the platform is obtained through four spherical joints that are placed along the axis $\left(O_{p}, \mathbf{x}_{p}\right)$ and $\left(O_{p}, \mathbf{y}_{p}\right)$ to insure the relative rotational DoFs between the two parts of the platform (see Fig. 5). Therefore, this design employs only spherical joints.

The legs were made of carbon fiber tubes of $4 \mathrm{~mm}$ in 
diameter and $80 \mathrm{~mm}$ in length. This choice was made in order to lighten the weight of the structure and to improve its strength in traction-compression. To control the manipulator, eight stick-slip Smaract actuators have been used. Each actuator is able to insure a repeatability of $\pm 1 \mu \mathrm{m}$ within an $18 \mathrm{~mm}$ travel motion.

The gripper fingers attached to the configurable platform have been designed to be replaceable depending on the required operation.

\section{Experimental VALidATION}

The demonstrator was designed to perform dexterous manipulation operations in confined environment. As an application, we decided to manipulate a screw in all DoFs and to insert it using two different modes.

\section{A. Experimental setup and preliminary tests}

An experimental prototype was fabricated and controlled. The configurable platform was made using a 3D printing machine. It was designed to attach two fingers of a tweezer to handle objects. The purpose of this prototype is to validate the proposed robotic structure through grasping, manipulating and inserting a screw. Hence, the manipulator was controlled using a joystick where the inverse kinematic model was used to calculate the displacement of the actuators for a given pose of the platform as illustrated in Fig. 6. The dimensional parameters presented in Table I were used for the control of the manipulator.

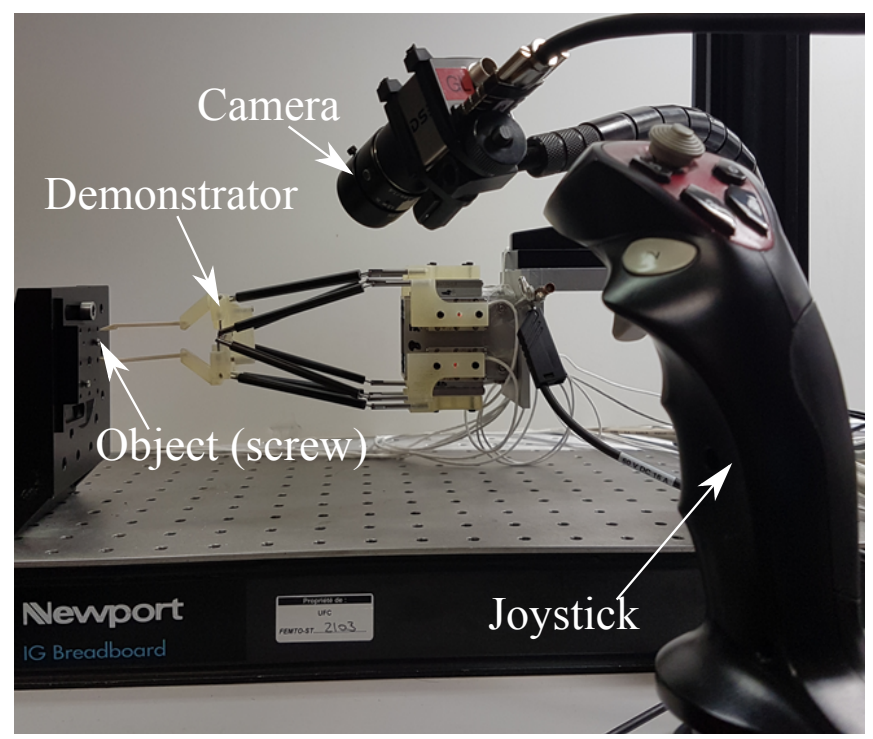

Fig. 6. Experimental setup.

\section{B. Screwing task}

To show the capabilities of this manipulator, a 2-mm (in diameter) screw is manipulated. First, both fingers grasp the screw and begin to unscrew it using a rolling movement between the two parts of the platform. Then, the screw is detached from the mechanical thread and positioned above the second thread. Finally, the object is oriented and screwed in the thread. The two modes of screwing (twist and yaw rotation) are presented in Fig. 7 and 8. This demonstration can be further appreciated in the video clip accompanying this paper.

These experiments validate the ability of the manipulator to realize dexterous tasks in a compact design thanks to the actuators located on the base. The trajectories of the end-effector were introduced via the joystick through the Cartesian coordinates and transformed to joint space trajectories using the inverse kinematics.

\section{CONCLUSION AND OUTLOOK}

In this paper, we presented a new class of parallel robot with a 2-DoF configurable platform and their practical implementation for two-fingered dexterous manipulation. The inverse kinematic and the jacobian matrix of an 8 DoF parallel manipulator was established.

A first prototype was realized and controlled via a joystick. This manipulator was teleoperated to unscrew, manipulate and screw a $2-\mathrm{mm}$ (in diameter) screw which demonstrates the dexterous capability of the proposed concept.

Future works will focus on the miniaturization of the proposed structure with a design optimization to employ it for micro and nano manipulations inside a scanning electron microscope (SEM).

\section{ACKNOWLEDGMENTS}

This work was supported by the Région de BourgogneFranche-Comté, Equipex ROBOTEX project (ANR-10EQPX-44-01) and the French "Investissements d'Avenir" program, project ISITE-BFC Robotics Nanofactory (ANR-15-IDEX-03). The authors gratefully acknowledge Patrick Rougeot, Gérard Michel and Martial Personeni from FEMTO-ST Institute for their technical assistance.

\section{REFERENCES}

[1] J.-P. Merlet, Parallel robots. Springer Science \& Business Media, 2006, vol. 128.

[2] F. Pierrot and O. Company, "H4: a new family of 4-DoF parallel robots," in 1999 IEEE/ASME International Conference on Advanced Intelligent Mechatronics, 1999, pp. 508-513.

[3] R. Clavel, "Device for the movement and positioning of an element in space," Dec. 11 1990, US Patent 4,976,582.

[4] H.-B. Choi, O. Company, F. Pierrot, A. Konno, T. Shibukawa, and M. Uchiyama, "Design and control of a novel 4-DoF parallel robot H4," in IEEE International Conference on Robotics and Automation, vol. 1, 2003, pp. 1185-1190.

[5] V. Nabat, M. De La O Rodriguez, O. Company, S. Krut, and F. Pierrot, "Par4: Very high speed parallel robot for pick-andplace," in IEEE/RSJ International Conference on Intelligent Robots and Systems (IROS), 2005, pp. 1202-1207.

[6] B.-J. Yi, H. Y. Na, J. H. Lee, Y.-S. Hong, S.-R. Oh, I. H. Suh, and W. K. Kim, "Design of a parallel-type gripper mechanism," The International Journal of Robotics Research, vol. 21, no. 7, pp. 661-676, 2002.

[7] A. G. Hoevenaars, P. Lambert, and J. L. Herder, "Kinematic design of two elementary 3-DoF parallel manipulators with configurable platforms," in Computational Kinematics. Springer, 2014, pp. 315-322.

[8] X. Kang and J. S. Dai, "Relevance and Transferability for Parallel Mechanisms With Reconfigurable Platforms," Journal of Mechanisms and Robotics, vol. 11, no. 3, p. 031012, 2019. 

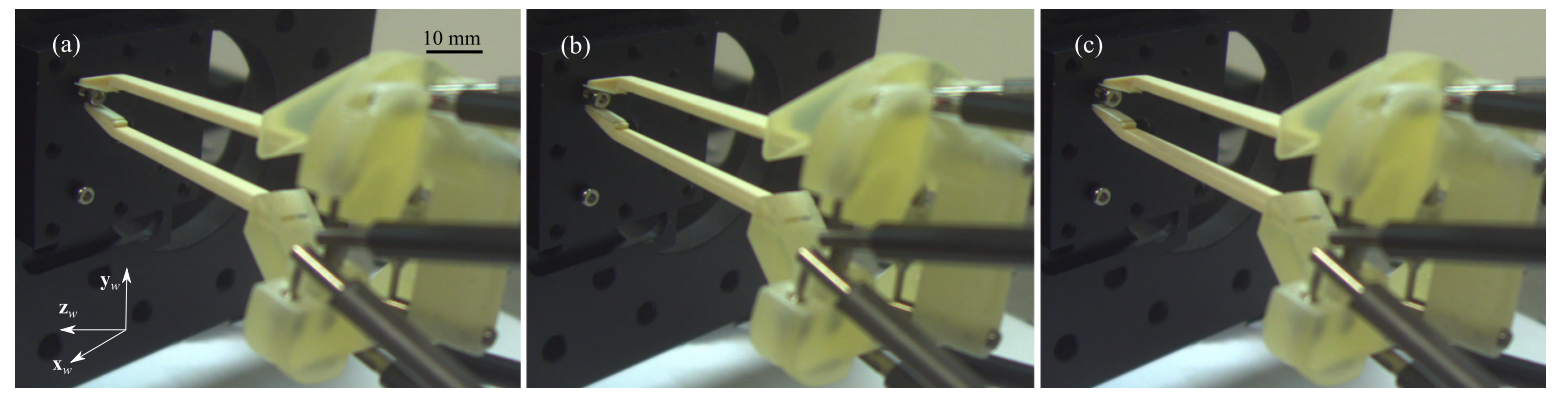

Fig. 7. Screwing a 2-mm screw using the twist mobility between the two fingers.
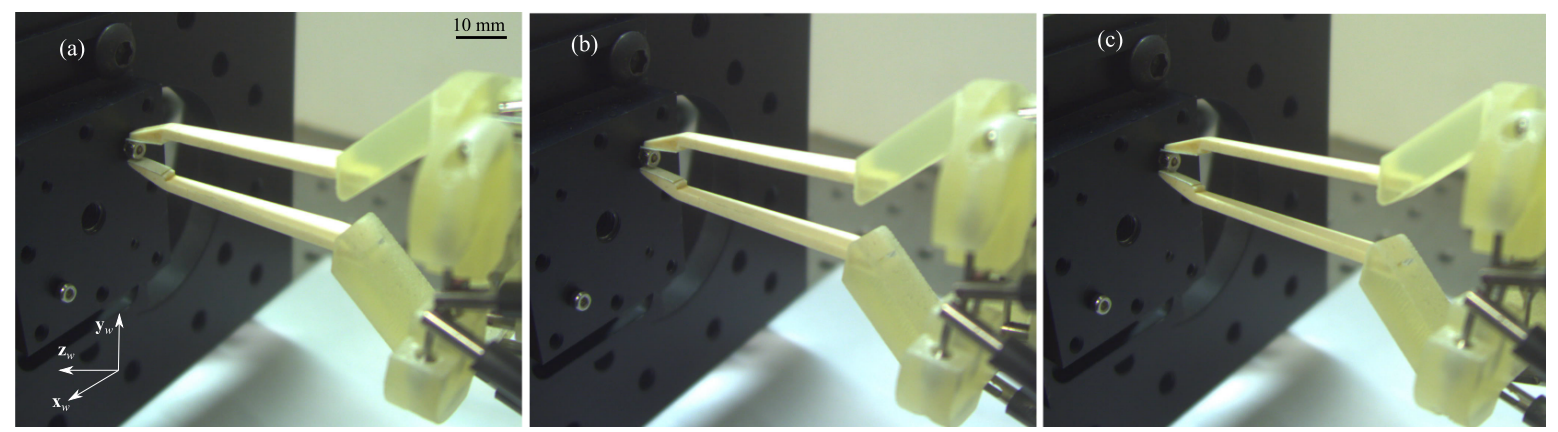

Fig. 8. Screwing a 2-mm screw using the yaw rotational mobility of the whole platform.

[9] P. Lambert, H. Langen, and R. M. Schmidt, "A novel 5DoF fully parallel robot combining 3T1R motion and grasping," in ASME International Design Engineering Technical Conferences and Computers and Information in Engineering Conference, 2010, pp. 1123-1130.

[10] P. Lambert and J. Herder, "A novel parallel haptic device with 7 degrees of freedom," in 2015 IEEE World Haptics Conference (WHC), 2015, pp. 183-188.

[11] M. Isaksson, C. Gosselin, and K. Marlow, "An introduction to utilising the redundancy of a kinematically redundant parallel manipulator to operate a gripper," Mechanism and Machine Theory, vol. 101, pp. 50-59, jul 2016.

[12] K. Wen, D. Harton, T. Lalibert, and C. Gosselin, "Kinematically Redundant (6+3)-DoF Hybrid Parallel Robot with Large Orientational Workspace and Remotely Operated Gripper," in IEEE International Conference on Robotics and Automation, 2019, pp. 1672-1678.

[13] W. Haouas, R. Dahmouche, N. Le Fort-Piat, and G. J. Laurent, "4-DoF spherical parallel wrist with embedded grasping capability for minimally invasive surgery," in 2016 IEEE/RSJ International Conference on Intelligent Robots and Systems (IROS), 2016, pp. 2363-2368.

[14] W. Haouas, R. Dahmouche, and G. J. Laurent, "Analysis of an integrated 4-DoF parallel wrist for dexterous gripping," in 2018 IEEE 14th International Conference on Automation Science and Engineering (CASE), 2018, pp. 1448-1453.

[15] W. Haouas, R. Dahmouche, N. Le Fort-Piat, and G. J. Laurent, "A new seven degrees-of-freedom parallel robot with a foldable platform," Journal of Mechanisms and Robotics, vol. 10, no. 4, p. 045001, 2018.

[16] W. Haouas, R. Dahmouche, J. Agnus, N. Le Fort-Piat, and G. J. Laurent, "New integrated silicon-PDMS process for compliant micro-mechanisms," Journal of Micromechanics and Microengineering, vol. 27, no. 12, pp. 2-6, aug 2017.

[17] J. A. Seon, R. Dahmouche, B. Brazey, and M. Gauthier, "Finger trajectory generation for planar dexterous micromanipulation," in IEEE International Conference on Robotics and Automation (ICRA), vol. 2016-June, 2016, pp. 392-398.

[18] B. Brazey, R. Dahmouche, J.-A. Seon, and M. Gauthier, "Experimental validation of in-hand planar orientation and translation in microscale," Intelligent Service Robotics, vol. 9 , no. $2,2016$.
[19] J. A. Seon, R. Dahmouche, and M. Gauthier, "Enhance InHand Dexterous Micromanipulation by Exploiting Adhesion Forces," IEEE Transactions on Robotics, vol. 34, no. 1, pp. 113-125, feb 2017. 